\title{
EL ASCENSO A DIOS EN EL DE MUSICA DE SAN AGUSTIIN DE HIPONA
}

\section{The Ascension to God in the De Musica of Saint Agustin of Hippo}

\author{
Jean Luis Arana Alencastre \\ Pontificia Universidad Católica del Perú, Perú \\ https://orcid.org/0000-0003-0910-7106 \\ Sobrio64@hotmail.com
}

\begin{abstract}
RESUMEN
La búsqueda de Dios ha sido una de los principales problemas en la filosofía del mundo antiguo. Y la pregunta por Dios, entendido como principio, a su vez, ha sido una o la cuestión más importante e interesante entre los clásicos de la antigüedad. Entre las concepciones sobre Dios, la de Agustín es la de considerarlo como un Dios personal y como el Ser. Y en su libro De Musica se refleja un camino epistemológico a través del ritmo hacia las armonías eternas, las cuales están en el Ser. Entonces, ¿cómo sería posible el ascenso del alma? ¿Qué vía emplea Agustín? Este artículo trata de explicar estas dos cuestiones. En primer lugar, nos aproximaremos a la noción de ascenso en Agustín. Y, en segundo lugar, cómo ese ascenso se puede dar a través del ritmo hacia las armonías eternas.
\end{abstract}

\section{Palabras clave:}

Ritmo, música, armonía, Ser, Dios, creación, camino, iluminación. Interior, interioridad, vía, eterno, temporal, ley, número.

\begin{abstract}
The quest for God has been a major one in the ancient world. And the question about God as Principle, one of the most important and interesting among the classics. Among the conceptions of God, the Augustinian one considers him as a personal God and as the Being itself. In his De Musica, there is an epistemological path through the rhythm to the eternal harmonies which are in the Being. How is the ascent of the soul possible? Which path did he indicates? This article tries to explain both subjects. First, we will analyze the ascent in Augustine and then how that ascent is possible through rhythm to the eternal harmonies.
\end{abstract}

\section{Keywords:}

Rhythm, music, harmony, Being, God, creation, path, illumination. Interior, interiority, way, eternal, temporal, law, number. 


\section{La vía agustiniana: el interior}

Para Agustín la filosofía, como para Pitágoras o Platón, es un estudio o amor a la sabiduría. No se trata sólo de una definición nominal, sino real; es decir no es sólo un estudio especulativo de las causas últimas o de la realidad fundamental, como decía Aristóteles, sino una búsqueda existencial que tiene como fin la propia realización y la vita beata, como lo sostenían Platón, Cicerón y Plotino: la recta vía de la vida es llamada sabiduría (recta via vitae sapientia nominatur) (Contra Academicos 1, 2, 5).

Las vías que existen para llegar a la verdad, que sería un atributo de Dios, son varias: la mayéutica de Sócrates, la dialéctica de Platón y la lógica de Aristóteles y la de los Estoicos. San Agustín conoce los tres métodos de la antigüedad; $y$, sin embargo, abre una nueva vía totalmente propia: la vía de la interioridad o de la introspección, que consiste en buscar la verdad mirando hacia el propio interior: "no busques fuera, sino que en el hombre interior habita la verdad" (Noli foras ire; in interiore homine habitat veritas) (De vera religione 39, 72). Es allí donde el ser humano podrá buscar a Dios, puesto mora en el interior de toda persona. En ese sentido, mientras que en la mayéutica y en la dialéctica donde se busca la verdad dirigiéndose a otro, Agustín la busca dirigiéndose a sí mismo, e interrogándose encuentra la respuesta dentro de sí, pues Dios es la Verdad. Es así que, Agustín parte de sí mismo; pues piensa que encada uno de nosotros se encuentra la Verdad en alguna medida.

Esto es, Agustín excluye que la verdad sea inferior a nuestra mente, pues hacemos juicios a partir de ella y no sobre ella, por lo que la Verdad tendría que ser superior a nuestra mente para que podamos emitir los juicios. Es a partir de ello que, el mirar atentamente dentro de uno mismo permita descubrir que hay en el interior mucho más de lo que debería tener por la sola naturaleza finita, frágil y mudable de uno mismo. Esto es cada persona tiene en sí misma la Verdad, la Bondad, la Belleza, la Libertad. Las cuales son perfecciones que están en él, pero que no son suyas, es decir, no le pertenecen plena y definitivamente. Pues no puede disponer de ellas sino solo recibirlas como parte de su ser más íntimo. Por tanto, son de aquel que es la Verdad, la Belleza, la Bondad y la Libertad por esencia: Dios.

Dios que, además concibe como el Ser. Por lo tanto, conocerse a sí mismo es conocer a Dios: Quien se entrega a la percepción de las cosas sensibles, no sólo está alejado de Dios, más aún de sí mismo (...) El sabio está ciertamente con Dios, porque también a sí mismo se entiende el que lo es (Quisquis ea soli novit quae corporis sensus atingit, non solum cum Deo esse non mihi videtur, nec secum quidem (...). Sapiens prorsus cum Deo est, nam et seipsum intelligit sapiens) (De ordine $2,2,5$ ).

Entonces, en Agustín, interioridad y metafísica no son dos métodos distintos, sino dos momentos de un único procedimiento, de un único método: la verdadera interioridad se da solamente cuando se extiende y se integra en la metafísica.

En sus obras de madurez, San Agustín continúa utilizando la vía de la interioridad, dentro del horizonte teológico, para conseguir una comprensión más profunda de la fe cristiana: el misterio de Cristo, como del misterio del pecado, del misterio de la Trinidad como del misterio de la 
gracia. En su Comentario al Evangelio de San Juan escribe lo siguiente:

Reconoce en ti mismo lo que está dentro de ti. Desnúdate no sólo de la vestidura sino también de la carne; entra dentro de ti mismo; penetra en tu vestíbulo secreto, tu mente. Si estás lejos de ti mismo, ¿cómo puedes acercarte a Dios? Pues no en el cuerpo, sino en la mente, es como el hombre ha sido creado a imagen de Dios. En su imagen buscamos descubrir a Dios" (In Ioh. Ev. 23, 10).

En el comentario a los Salmos escribe:

Hermanos, considerad atentamente lo que se encuentra en el alma del hombre. Por sí misma no posee ni luz ni potencia; por su propia cuenta no es sabia ni fuerte, ni es luminosa por sí misma, ni es virtuosa por sí misma. Hay una fuente y un origen de la virtud, hay una raíz de la sabiduría, hay — si es lícito llamarla así - una razón de la verdad inmutable, alejándose de la cual el alma tropieza en las tinieblas, mientras que, si se acerca a ella, el alma se llena de luz" (In Ps. 58, Srm. 1, 18).

Y vuelve sobre la misma idea en las Retractaciones:

En lo que se refiere a la naturaleza del hombre, no hay cosa mejor que su mente o su razón. Pero quien desee vivir felizmente, no debe vivir según la mente, porque así viviría según el hombre, mientras que para poder alcanzar la felicidad se debe vivir según Dios. Por lo tanto, la mente, para alcanzar su felicidad no debe tender a sí misma sino someterse a Dios" (Retract. 1, 1, 2).

\section{El ascenso hacia Dios: la anábasis}

En sus Confesiones, Agustín de Hipona (7.16) describe el ascenso con estas palabras:

Movido por esos [libros platónicos] a retornar a mí mismo, contigo como mi guía entré en lo profundo de mi ser; fui capaz de hacerlo desde que Tú te convertiste en mi ayudador [Salmo 29: 11]...Cuando por primera vez vine a conocerte a Ti, Tú me levantaste para que yo pueda ver que lo que veía es...".

Es decir, en primer lugar, Dios se une a Agustín para ser su guía. Esto es algo extraordinario dice Mann-; pues cómo podríamos imaginarnos a la Idea de la Belleza misma acompañando a Platón, o el Uno siendo el asistente de Plotino. En segundo lugar, Agustín es elevado (adsumpsisti); es decir, no depende de él llegar a las alturas de lo divino, sino que es llevado. Esto, sin embargo, no es automático, es necesario que aquel que quiera subir deba estar preparado para ello, para ver algo de lo divino. Este aspecto Agustín lo elaborará mejor posteriormente en su doctrina de la gratia, y en la Confesiones constantemente estará refiriéndose a esta ayuda de Dios. Sin embargo, ver a Dios no tiene el mismo matiz que en Platón, pues para Agustín Dios trasciende radicalmente lo finito. Y solo podríamos admirarlo desde nuestra perspectiva limitada y finita (Mann, 2014). Y, aun así, nuestro objetivo es amar y admirar lo divino (Confesiones 7.16).

Para Miranda, mientras que la relación plotiniana del Uno con el alma es impersonal; es decir indiferente y universal, en Agustín el "principio fundamental de la ascensión del hombre es que Dios es relación en sí mismo, 
que, a su vez, fundamenta el encuentro con las criaturas" (Miranda, 2008). De esa manera, para Agustín, Dios es el Ser, Pensamiento y Amor como puede habérselo sugerido la lectura del Idipsum (Cf. Salmo 121. 5), el cual pudo ser leído en relación al Éxodo 3, 14 (Miranda, 2008). Y aunque Porfirio pudo influir en su pensamiento, ni en Porfirio ni en Plotino se encuentra la idea de que Dios va al encuentro del hombre. Por tanto, si es importante para Agustín el verso Éxodo 3, 14: "Dios dijo a Moisés: "Yo soy el que soy". Y añadió: "Así dirás a los israelitas: "Yo soy" me ha enviado a vosotros". También lo es el verso 15 del mismo libro donde dice: "Siguió Dios diciendo a Moisés: "Así dirás a los israelitas: Yahveh, el Dios de vuestros padres, el Dios de Abraham, el Dios de Isaac, el Dios de Jacob, me ha enviado a vosotros. Este es mi nombre para siempre, por él seré invocado de generación en generación". Lo que significa que al ser solo se le comprende siempre en relación con la misericordia; es decir el nomen substantiae es comprendido con el nomen misericordiae. Y entonces, se aleja Agustín de Plotino, con Porfirio, y de este tomando a Dios como Aquel que se ha hecho hombre.

Asimismo, Nussbaum, dice que el ascenso agustiniano supera en un sentido al platónico-plotiniano: en la humilitas. Y es que el ascenso platónicoplotiniano esconde la creencia de que por el propio esfuerzo los hombres pueden hacerse semejantes a los dioses. Pero esa autosuficiencia hace que rechacen la compasión; pues sería algo contaminado por el mundo. Y eso a su vez es lo que no permite reconocer la necesidad y el sufrimiento de otros (Nussbaum, 2008). La vía humilitas es condición, en cambio de salvación al reconocerse incompleto e imperfecto.
De igual manera para Harrison, el ascenso de Agustín es posible por la providencia y gracia de Dios en crear, ordenar y mantener su creación para guiar a los hombres de vuelta a Él, así: "Pues, la verdadera religión es aquella por la cual el alma es unida con Dios pues así se une de nuevo por la reconciliación con Él..." (Harrison, 2006, p. 51).

Es cierto que Agustín tiene influencia de Platón, Plotino y Porfirio en cuanto al ascenso del alma para contemplar las verdades eternas, como no tenerla; sin embargo, su pensamiento no se limita ni se reduce a de ellos. El ascenso en Agustín tiene un matiz muy diferente al de todos ellos.

Entonces, podemos decir que en Agustín se muestra una diferencia sustancial con Platón, Plotino y Porfirio: que Dios es el camino de nuestro ser, y sobre todo la posibilidad de conocer y amar (De civitas Dei 11.28); pues es el primer impulso que posibilita nuestra subida. Por ello, la primacía del amor es fundamental y acto primero en la contemplación de lo divino. Así, el amor viene primero desde el punto de vista epistemológico también.

La caridad es la única condición para una vida cognitiva armoniosa. Solo la caridad puede restaurar el conocimiento y permitir a los filósofos a rendirse al mandato que resume la empresa filosófica como un todo, llamada "Conócete a ti mismo" (Gioia, 2008, p. 301).

En ese sentido "No es simplemente una cuestión de entender una verdad desde un punto de vista de pura especulación, sino de acceder a ella "con toda el alma" (Zum, 1992, p. 4). Y para acceder a ella es necesario la dilectio: "The trigger is love itself (God)" (Gioia, 2008, p. 183). Por ello 
es sumamente importante profundizar en la noción de dilectio como lo primero necesario para conocer lo divino en san Agustín. Lo cual vemos reflejado en estas palabras del Obispo de Hipona:

Nadie diga: "No sé qué amar." Ame al hermano y amará al amor. Mejor conoce la dilección que le impulsa al amor que al hermano a quien ama. He aquí cómo puedes conocer mejor a Dios que al hermano; más conocido porque está más presente; más conocido porque es algo más íntimo; más conocido porque es algo más cierto. Abraza al Dios amor y abraza a Dios por amor [Ecce iam potest notiorem deum habere quam fratrem, plane notiorem quia praesentiorem, notiorem quia interiorem, notiorem quia certiorem. Amplectere dilectionem deum et dilectione amplectere deum] (De Trinitatde 8.12).

Así, Dios es amor y es conocido en el grado más alto posible porque él es la cosa más íntima, él es la cosa más presente, él es la cosa más cierta. Este amor es ambos la forma que conocemos-por decirlo así- y por lo cual esta forma nos transforma y nos informa (Gioia, 2008).

Ahora bien, en sus Confesiones, Agustín describe su ascenso desde lo mudable hacia lo inmutable,

arrebatado hacia ti por tu hermosura, era luego apartado de ti por mi peso... Porque buscando yo de dónde aprobaba la hermosura de los cuerpos-ya celestes, ya terrestres-y qué era lo que había en mí para juzgar rápida y cabalmente de las cosas mudables cuando decía: «Esto debe ser así, aquello no debe ser así»; buscando, digo, de dónde juzgaba yo cuando así juzgaba, hallé que estaba la inconmutable y verdadera eternidad de la verdad sobre mi mente mudable.

Y fui subiendo gradualmente de los cuerpos al alma, que siente por el cuerpo; y de aquí al sentido íntimo, al que comunican o anuncian los sentidos del cuerpo las cosas exteriores, y hasta el cual pueden llegar las bestias. De aquí pasé nuevamente a la potencia raciocinante, a la que pertenece juzgar de los datos de los sentidos corporales, la cual, a su vez, juzgándose a sí misma mudable, se remontó a la misma inteligencia, y apartó el pensamiento de la costumbre, y se sustrajo a la multitud de fantasmas contradictorios para ver de qué luz estaba inundada, cuando sin ninguna duda clamaba que lo inconmutable debía ser preferido a lo mudable; y de dónde conocía yo lo inconmutable, ya que si no lo conociera de algún modo, de ninguno lo antepondría a lo mudable con tanta certeza. Y, finalmente, llegué a «lo que es» en un golpe de vista trepidante. Entonces fue cuando «vi tus cosas invisibles por la inteligencia de las cosas creadas»; pero no pude fijar en ellas mi vista, antes, herida de nuevo mi flaqueza, volví a las cosas ordinarias, no llevando conmigo sino un recuerdo amoroso y como apetito de viandas sabrosas que aún no podía comer (Confesiones VII. 17. 23).

Agustín dice "vi tus cosas invisibles por la inteligencia" no dice te vi. En ese sentido, puede pensarse que el intelecto 
no es suficiente para contemplar a Dios, sino que para entrar en la realidad del ser es necesario establecer la convergencia de la percepción sensible con la razón que se da en el acto del intelecto junto con en el despliegue de la modulación en el lenguaje, pues es dentro de este que se gesta la unión del ser.

Y, por esto, es central considerar lo que Agustín dibujó en la tradición cultural y filosófica, aquello que pudo compartir con sus contemporáneos paganos: la poesía. Pues esta es lenguaje, un tipo de lenguaje que se despliega en los ritmos y significa no solo a partir de sus conceptos sino también desde la música. Aunque no tuvo la misma lectura que ellos. Es así que escribió,

...no pensamos emprenderlo por otra razón alguna sino para que los jóvenes, y aun las personas de cualquier edad, a quienes dotó Dios de buena inteligencia, se arrancaran, bajo guía de la razón, no precipitadamente, más por modo escalonado, de los sentidos de la carne y de las literaturas carnales a las que no es difícil apegarse, y por amor a la verdad inmutable quedaran fijos en el único Dios y Señor de todas las cosas que, sin mediación de criatura alguna, dirige las almas humanas (De musica VI. 1).

Y es que el cuerpo, para Agustín, no solo está animado por el alma sino, además, bajo la finalidad de aquel que lo creó. Y el alma disponiendo de y en aquel por designio divino puede elevarse por medio de la modulación en el lenguaje más allá del mero existir al existir realmente, es decir, al Ser. Lo cual es un camino como respuesta a la inquietud ontológica de todo hombre: el desear la unión con el
Ser; pues viene del Ser. En ese sentido, las cosas corpóreas y los signos exteriores no causan la existencia verdadera por sí mismas o por el hecho de percibirlas o ejecutarlas, sino que es la voluntad quien hace uso de ellas en camino hacia el Ser y al momento de moverse generan la imagen del Ser, y en ese sentido podría decirse que hay una especie de unión. De esa forma, son signos que ayudan a revelar lo profundo e inescrutable de la voluntad del Ser.

Finalmente, la revelación del Ser es gracia; pues es otorgada y por ella es que se concede la participación en el Ser, a través de la modulación, solamente a aquel virtuoso y amante, que busca el ser por sí mismo y no por otra cosa. Esta gracia se manifiesta en la vivencia de la existencia que vislumbra lo real desde lo temporal, como siendo un acontecimiento, es decir, un acontecimiento que transcurre él mismo durante la modulación. Desde ese aspecto se crea algo donde antes no había nada. Y no por mucho esfuerzo es que se entra ni por la comprensión intelectual solamente sino por cierta disposición del corazón ante el camino, ante el Ser.

\section{El camino de la interioridad}

Después de escribir sus Confesiones Agustín vuelve al De musica para completarlo con el libro VI en el cual explica el camino hacia la verdad inmutable, camino que ya había trazado desde los libros anteriores en compañía de los gramáticos y los poetas. Y un camino que, sin duda, es necesario hacia la verdad (De musica VI. 1.1).

El diálogo De música fue escrito en el 387 en Milán y terminado años después en África. En dicho diálogo se muestra algo nuevo: el despliegue de un largo material para explicar el entendimiento 
interior. En él, los conceptos de armonía y ritmo que dan forma a la realidad y a nuestro conocimiento son el objeto de la búsqueda agustiniana (Parodi, 2011). Y es precisamente en la vuelta a la práctica y al origen sensible de la precepción del ritmo donde puede encontrarse algo más de la profundidad que envuelve el pensamiento de Agustín.

Escucha esto: pienso que alguna vez tú habrás observado obras o artesanos de este género que golpean con el escardillo o con el hacha siempre el mismo sitio y conducen el golpe a donde indica el propio espíritu, y cuando nos arriesgamos a imitarlos intentando hacer [lo mismo], a menudo somos por ellos burlados ${ }^{1}$ (De Musica I. 4. 9).

El ritmo no es fácil de conquistar y el mismo aun nos indica la dirección del propio espíritu y su modulación, pues esta se comprueba en el tiempo de una precisa marcación de los golpes. Dicha búsqueda es una especie de representarse la poca claridad y el poco dominio de nuestros horizontes, pues al remontarse "desde la consciencia de la sensación a la profundización de sus mecanismos, los datos característicos de la sensibilidad vuelven a poner en discusión constantemente, los puntos conquistados" (Parodi, 2011, p. 34) o aparentemente conquistados.

Ahora bien, el movimiento hacia el interior tendrá lugar también en la búsqueda por las armonías eternas del De Musica. Dicho movimiento será también el que Agustín empleará para poder responder a la pregunta: ¿Cómo podemos conocer las armonías eternas? ¿Qué misterioso poder tiene la música para servir de vehículo a la gracia divina y suscitar una conmoción espiritual profundísima? Sin duda que en el De Musica se puede ver el movimiento que pretende un ascenso a través del interior: en la conmoción del espíritu y en el pensamiento de los números.

En el libro VI del De musica, se muestra de una forma particular una compleja interrelación entre el conocimiento sensible y el cocimiento intelectual. Y se hace mediante aspectos objetivos representados en la materialidad del sonido que serían los números sonoros; mediante aspectos subjetivos representados en la actividad mental que organiza la estructura espacio-tiempo; y, finalmente, mediante representaciones que se regulan, en primera instancia, por la memoria, y luego por el juicio (Parodi 2001).

En aquel libro, Agustín nos lleva a descubrir la belleza dada por el ritmo no solo en la correspondencia de relaciones y proporciones, que se basa ciertamente en la subjetividad de la consciencia, sino también en la igualdad y la unidad en sí, a las cuales se llega, avanzando desde la exterioridad a la interioridad y de allí, a lo superior. Por ello es importante analizar el camino epistemológico que traza Agustín hacia el interior del alma, el cual permitiría el paso de los ritmos a las armonías y finalmente al Ser. Aunque, el camino hacia el ser no puede ser solamente una comprensión intelectual, sino un gozo de esa misma realidad, una anticipación de la Verdad, del Bien y de la Belleza lo cual era

1 Attende etiam istud: Nam opinor non nanquam te animadvertisse fabros, vel huiusmodi opifices, ascia sive securi eundem locum feriendo repetere, et non alio cuam eo quo intendit animus ictum perducere; quod nos tentantes cum assecui nequimus, ab eis saepe irridemur. 
en lo que justamente consistía la felicidad de la vida eterna (Carry, 2008).

El objetivo del De Musica, como lo expone Agustín, es llegar a contemplar las armonías eternas y para ello el camino es hacia el interior:

La música tiene una única tarea primordial (...) disociar a la razón, mediante sus perfectas relaciones acústico-matemáticas, de los sentidos carnales y elevarla a la verdad inmutable, al único Dios y Señor de todas las cosas (...) Así pues, debe conducir al alma a reconocer que las cosas terrenas están subordinadas a las celestes (De Musica p, 343)

Es el alma quien reconoce aquellas verdades inmutables. Así, de acuerdo a ello, en el libro de las Revisiones I, 11, Agustín escribe refiriendo al sexto libro del De Musica donde trata de "cómo de los números corporales y espirituales, pero mudables, se llega a los números inmutables, que están ya en la misma verdad inmutable, y de este modo las cosas invisibles de Dios se hacen inteligibles por las cosas que han sido hechas visibles" lquomodo a corporalibus et spiritualibus, sed mutabilibus numeris, perveniatur ad immutabiles números] (Retract. I. II. 1). Es importante notar que, para Agustín, en la música se encuentra algo central para llegar a las cosas invisibles de Dios por medio de lo visible. El hombre entonces será excelente no haciendo cosas bien medidas sino vislumbrando la naturaleza de los números en su mismo interior, en su inteligencia [Nono ergo numerosa faciendo, sed números cognoscendo melior sum] (De Ordine. II 49). Es decir, conociendo de la teoría numérica en donde se halla la verdadera forma de la música.
Así, en primer lugar, Agustín define a la música como la ciencia del modular bien (De Musica I. 2.2). Esta definición se adscribe a la que da Varro: música es la ciencia del modular bien (Musica est scientia bene modulandi) (De Musica II.2.2). Así mientras que, Agustín parte de Cicerón para cuestiones sobre la certeza y la vida feliz, mientras toma, en parte, los principios de fuentes neoplatónicas en torno al ascenso del alma desde la percepción sensible a la razón y a través del orden de las disciplinae, el cual se identificaba también con la providencia de Dios como último objeto de estudio, su interés con respecto al lenguaje, a la gramática y a la dialéctica, refleja las investigaciones de Varro, quien había investigado la cuestión sobre cómo y por qué palabras específicas eran impuestas a las cosas y a los conceptos inquiriendo sobre el origen, el cambio en el tiempo y la inflexión en las palabras.

Ahora bien, el método que emplea para conocer Agustín en su libro De Musica es el de la dialéctica, pero con un matiz especial, la inclusión de los sentidos en el decurso del movimiento; a esta nueva dialéctica la llamaré dialéctica musical. La cual permitirá entrar en lo íntimo para poder realizar el ascenso hacia las realidades eternas, con ayuda del Maestro, quien puede representar a Dios mismo yendo al encuentro del hombre; y haciéndolo ver en su inteligencia los números y las armonías. Así, se establece un movimiento dialéctico que intenta versar entre la emoción por medio de los sentidos y la razón por medio del pensamiento.

Agustín mismo explica el método a seguir, pues hace que el Maestro le dice al Discípulo: "Lo haré, o más bien lo harás tú. Porque yo no haré otra cosa que preguntarte e inquirirte todo; tú, en 
cambio, con tus respuestas vas a explicar todo, como quiera que ello sea, y que tú pareces indagar como si lo desconocieses ahora" (De Musica Liber I 15). Es así que el Maestro sugiere que es el Discípulo quien hará el esfuerzo de explicar, sin embargo, recibirá ayuda, la guía de Aquel que ya sabe el camino. Además, no solo será un ejercicio dialéctico de la razón sino también del gusto, como vemos en el siguiente pasaje:

Pero esto ha sido por efecto de mi pronunciación, a saber: por el vicio que los gramáticos llaman barbarismo; porque primus consta de sílaba larga y breve, mientras primis tiene dos largas. Pero yo hice breve la última. Así no han sufrido engaño tus oídos. Por lo cual vamos a probar repetidas veces si percibes en mi pronunciación qué es tiempo largo y tiempo no largo en las sílabas, para que nuestra discusión, a base de mis preguntas y tus respuestas, como dispusimos, pueda seguir adelante. Así voy a repetir ahora el mismo verso en que había cometido un barbarismo, y aquella sílaba que hice breve, para que no se molestaran tus oídos, la alargaré, como ordenan los gramáticos. Tú hazme saber si la medida de este verso causa placer alguno en tu sensibilidad. Vaya así, en efecto, mi pronunciación: arma virumque cano, Troiae qui primis ab oris (De Musica Liber I 27).

Los oídos serán los jueces de la certeza de la razón. Es interesante ver el énfasis en los sentidos; pues ellos confirmarán lo que el pensamiento les mostraba. Entonces, el ascenso es hacia el interior y que la dialéctica mostrada en el De Musica es una dialéctica musical que toma en cuenta lo sensible:
Y me maravillo de que aprecies como falsas las conclusiones que entre nosotros dejamos asentadas, pues nada hay más seguro que los números, nada mejor ordenado que este recuento y disposición de los pies. Porque desde la misma naturaleza intrínseca de los números, que en modo alguno yerra, brota como expresión lo que en ellos hemos distinguido que tiene poder para encantar los oídos y para ocupar el primer rango en el ritmo. Pero mejor experiméntalo tú al repetir yo muchas veces quae canitis sub antris y al acariciar tu sensibilidad con esta armonía. ¿Qué diferencia habría en ese verso si añadiese una breve a su final y si de igual modo lo repitiese así: quae canitis sub antrisve? (De Musica Liber IV 56).

El libro VI del De Musica empieza con la línea del Himno de Ambrosio que Mónica había recordado y cantado en Cassiciacum; y que él mismo había recordado una vez más cuando su madre estaba ya en su tumba en Ostia (Confesiones IX. 12; 32; X. 37-57).

Una cosa es, en efecto, no existir; otra, el hecho de que no pueda encontrarse, sea por hombre alguno, sea por nosotros. Mas yo pienso que, cuando se canta el verso que nosotros hemos citado: Deus creator omnium (Dios, creador de todas las cosas), lo estamos oyendo por medio de los números entendidos, lo reconocemos por los de la memoria, lo pronunciamos por los proferidos y sentimos placer gracias a los de juicio; y no sé a través de qué otros números lo valoramos; y partiendo de ese placer experimentado, que es 
como una decisión espontánea de los números de juicio, emitimos sobre dicho placer una sentencia mucho más firme, de acuerdo con esas armonías más ocultas (De Musica VI. 2. 2-4.7).

¿Cómo es que usando los sentidos del cuerpo es posible percibir de alguna manera la dulzura e igualdad de Dios? La dulzura e igualdad (aequalitas) material no puede aproximarse a la dulzura de Dios y, aun así, puede servir como indicador de que el ser nos dice que el Dios es dulce (Mac Cormack, 1998). Así, aquel verso Deus creator omnium:

No solo produce encanto sumo a los oídos por la armonía de su sonido, sino mucho más al alma por la exactitud y la verdad de su afirmación. Si es que no te turba la torpeza de quienes, para decirlo de modo más suave, niegan pueda nacer algo de la nada, cuando afirmamos que lo ha hecho el Dios omnipotente. ¿Es que el artista, por medio de las armonías racionales que hay en su arte, puede producir las armonías sensibles contenidas en su potencia habitual, y mueve, por las armonías sensibles, los números proferidos, con los que pone en acción sus miembros y a los que conciernen los intervalos de los tiempos, y por estas últimas armonías puede a su vez configurar de la madera unas formas visibles - armoniosas por su simetría espacial-, y la naturaleza de las cosas, obediente a las indicaciones de Dios, no puede producir la madera misma de la tierra y de los demás elementos, y Él no iba a poder producir de la nada estos últimos seres? (De Musica VI. 17. 57).

Los cuatro pies yámbicos ${ }^{2}$ de Ambrosio indican que existe una medida que está en la razón y permanece en la memoria independientemente de que sean pronunciados. Entonces, el sentido y la razón concuerdan en el entendimiento del verso Deus creator ómnium de la misma forma en que lo hacían los versos de Virgilio y los otros poetas. Lo que lo diferencia de ellos, no obstante, es que la afirmación Deus creator omnium permitía una mayor y más completa convergencia (concordantia) de la percepción sensible con la razón, una especie de vehículo entre la una y la otra que no es posible cuando una afirmación no es verdadera. Los pies, en concordancia, son una expresión corpórea del ser, que reflejan su belleza, creando así una realidad como autoexpresión, pues las cosas antes de que fuesen estaban en la mente de quien las creó, donde eran más perfectas y verdaderas, donde eran eternas e inmutables (De Genn ad Litt. V. 25. 33). Concordancia, lo que los griegos llaman armonía, es la compaginación de una cosa con otra, "es de valor sumo en el acorde orquestal y grandioso de la creación" (De Trinitate IV. 2. 4).

Y, tal vez, por ello se llama Universo, Uni-verso, Un-canto creado a imagen de Dios que se mueve en el tiempo desplegándose con armonía y con orden que refleja la ordenada sucesión del tiempo, la cual es descrita casi siempre por Agustín como una bella canción o un poema. Este orden manifiesta belleza que es mantenida, a pesar del mal, por Dios con providencia y justicia (De Natura Boni 36, 8; De Vera Religione 44, 5; De Libero Arbitrio III 26, 7; De Musica VI 2, 30; Salmo

2 Uno de los 28 pies rítmicos griegos que son usados tanto en la lírica como en la poética épica. 
7; 19). Es así que "el inmutable creador y moderador de las cosas mudables, conoce mucho mejor que el hombre lo que es oportuno en toda época, lo que en cada momento debe dar, añadir, eliminar, quitar, aumentar o disminuir para que la belleza del universo, de las que son particular las cosas a cada tiempo, se desarrolle y se cumpla como el concierto de un artista inefable" (Ep. 238i, 5). En ese sentido, todo acontecimiento es a la vez inevitable ${ }^{3}$, al menos en el sentido de su inminencia, es decir, de su posibilidad de realización. Esto es, desde su ya ser creado, es inevitable, pues es desde su posibilidad, y no en el sentido de que debe ser este ya determinado o que debía ser este ya determinado. Y, no obstante, Agustín propone la fascinante imagen de la historia al modo de un canto "cuyas oposiciones engendran armonías tan sublimes que solo Dios conoce su razón final, mientras que para el hombre permanecen cargadas de enigma (Alegría 1985). Donde todas las cosas son llamadas a la existencia desde la nada, y aunque el mal no sea nada, aún con su siniestro este universo es perfecto; por quien no hay absoluta disonancia porque el mal y el bien juntos armonizan (Soliloquia I. 2). Es en los Soliloquia que Agustín muestra una justificación estética del mal y una dependencia de la creación frente a Dios. Así, esta recibe la existencia moviéndose hacia Aquel, pues es Dios quien no solo ordena al mundo sino a la voluntad del hombre libre en la justicia que por medio de las leyes de Dios la voluntad se hace libre (Soliloquia I. 4); y se extingue moviéndose hacia la nada al alejarse del ser en la soberbia, en la creencia de su propia autosuficiencia. Aunque en realidad solo Dios puede ver y entender toda la sucesión temporal y el significado o sentido que pueden tener los eventos de la historia al poder unificarlos todos en Él (De Vera Religione 43; Confesiones XI. 29. 39).

El acto creativo de Dios tiene afinidad con la vía del arte; pues es quien produce una obra a partir de modelos interiorizados que están en Él mismo. Así, Dios, el poeta primero, el Verbo, los intuye al producir su obra de arte: "...ars ipsa per quam facta sunt omnia" (De libero arbitrio II. 15. 42). De esa manera, el Verbo es el artista primero (De Trinitate VI, 10,11), la causalidad propiamente ejemplar: "... exempla rerum, quas Plato appellat Ideas" (De Civitas Dei VII, 28). Mientras las Ideas son causas o "razones por las que todo fue hecho" (De libero arbitrio III. 5. 13), en Agustín razones yacen en Dios y no en otra parte. Es así como todo imita su belleza. Y el mundo conveniente y bueno del Génesis es también el producto constitutivamente bello. Entonces, Dios crea todo cuanto existe de la nada. Y mueve todo con armonía, pues todo cuando ha sido credo, existe por las armonías que están presentes en sus movimientos. Por tanto, el mundo, como obra, parte del Ser, que es Dios, y es creado a partir de las armonías eternas, las cuales empiezan a casua de la unidad (De musica VI. 17. 57).

Agustín muestra que todo lo creado contiene en sí mismo el ritmo donde yace la igualdad por la simetría de su composición. En ellos, se encuentra el toque divino de la armonía eterna. La medida de progresión de uno a cuatro, la igualdad de las partes que se halla en la longitud, en la anchura y en la altura viene de la correlación, es decir la analogía, "para que la anchura tenga respecto a la longitud la misma proporción que la longitud tiene respecto al punto indivisible

3 Esto conlleva un gran problema con respecto al devenir y a la libertad. Problema que la tesis no pretende abarcar, sino solo señalar o advertir. 
y la altura con relación a la anchura" (De Musica VI. 17. 58). De esa manera, todo ello viene del principio eterno de las armonías, de la semejanza, de la igualdad y del orden. Sin ellas, dice Agustín, la tierra nada sería.

Y ese principio de igualdad y de armonía eterna se encuentra en el interior del alma. Y la sensibilidad no puede estar en contradicción con la razón y es el oído quien percibe la armonía que se desprende de los números. Es decir, se inicia en la experiencia, y lo que se siente está de acuerdo con las armonías; pues lo oído es por medio de los números entendidos, lo reconocido por los de la memoria, lo pronunciado por los proferidos y sentimos placer gracias a los de juicio. Por ello dice que las cosas permanecen en una vida oculta en el secreto vientre de la naturaleza pueden salir abriéndose paso y ser creadas hacia fuera desarrollando de acuerdo a su propia medidas, números y pesos los cuales han recibido de Aquel que ha ordenado todo en medida, número y peso (De Trinitate III. 9. 26). Es así que, el Ser, el Dios de Agustín, le ha dado número incluso a las cosas más pequeñas y remotas, y todos los cuerpos tienen sus propios números, pero si los consideramos desde otro ángulo vemos que transcienden nuestras mentes y aceptan por ello la incambiable verdad (De Liberio Arbitrio II. 11. 32).

De esa manera se establece una relación entre las cosas del mundo que son percibidas y las armonías temporales que las preceden y están "ocultas y en silencio, y están dentro del movimiento" (De música VI. 17. 59), pues aquellas no pueden adquirir ni conservar las armonías locales que parecen estar en un modo de ser estable (I6ídem). Y, Agustín menciona el movimiento vital (vitalis motus) que precede a las armonías activas en los intervalos ordenados de los tiempos la cual obedece a Dios debido a una potencia que gobierna los tiempos. Es sobre esta potencia que las armonías racionales e intelectuales de las almas bienaventuradas y santas que, sin la mediación de ninguna otra naturaleza, recogen la ley misma de Dios (De Música VI. 17. 59). Así, el alma es capaz de recibir la ley eterna por medio de las armonías. Esta es la relación y el vínculo entre lo temporal y lo eterno. Y la vía para que el Ser se revele y entre en comunión con el alma es a través de la música, la cual se mueve en dichas armonías. En consecuencia, la posibilidad de del ascenso del alma a Dios se da por las armonías del alma y por lo ordenado mediante el número. Por tanto, la vía de la interioridad lleva el ascenso del alma hacia las armonías eternas y con ellas a la ley de la igualdad signo de Dios, el Ser, en ella.

\section{REFERENCIAS BIBLIOGRÁFICAS}

ALEGRÍA, C (1985). Música y palabra en el pensamiento de San Agustín sobre el tiempo. (Tesis de licenciatura, Pontificia Universidad Católica del Perú, Facultad de Letras y Ciencias Humanas. Lima, Perú)

AGUSTIN DE HIPONA (1956). Tratado sobre la Santísima Trinidad. Edición Bilingüe Tomo V. Primera edición española, introducción y notas del padre. Fr. Luis Arias O.S.A. Segunda Edición. Biblioteca de Autores Cristianos.

AGUSTIN DE HIPONA (1988). La Música. Biblioteca de autores cristianos.

AGUSTIN DE HIPONA (1988). Confesiones. Biblioteca de autores cristianos. 
MONDIN, B. (1996). Storia della Teologia. Edizioni Studio Domenicano, Bologna, (1), 377-393.

BOECIO (2012). Tratado de Música. [Trad. J. Picasso]. Fondo Editorial UCSS.

GIOIA, L. (2008). The Theological Epistemology of Augustine's De Trinitate. Oxford University.

HARRISON, C. (2005). Beauty and revelation in the thought of saint Augustine. Clarendon Press HARRISON, C. (2006). Rethinking Augustines Early Theology. University Press.

MANN, W. (2014). Augustine's Confesions. Philosophy in Autobiography. Oxford University Press.

MIRANDA, L. (2008). Ascensiones in corde. Interpretación bíblica y/o
Anábasis plotiniana. Estudio sobre el progreso espiritual en Agustín de Hipona. Gregorian \& Biblical Press.

NUSSBAUM, M. (2008). Paisajes del pensamiento. La inteligencia de la emociones. Paidós

PARODI, M. (2011). El paradigma filosófico agustiniano. Un modelo de Racionalidad y su

Crisis en el siglo XII. Meño y Dávila editores.

PLATÓN (1985). Diálogos I. Apología, Criton, Eutifrpon, Ion, Lisis, Carmides, Hipias menor, Hipias Mayor, Laques, Protágoras. (Introd. E. Lledó. Trad. M. Ángeles Durán y Francisco Lisi). Editorial Gredos.

ZUM, E. (1922). St. Augustine: being and nothingness in the Dialogs and Confessions. Parangon.

Fecha de recepción: 03/05/2021 Fecha de aceptación: 19/05/2021 
